\title{
Gradiente ambiental em comunidades arbóreas no baixo rio Jacuí
}

\section{Environmental gradient analysis in arboreal communities in the lower Jacuí river}

\author{
Marcelo Callegari Scipioni ${ }^{\mathrm{I}}$ Franklin Galvão $^{\mathrm{II}}$ \\ Solon Jonas Longhi"I Fabrício de Araújo Pedron ${ }^{I V}$
}

\section{RESUMO}

A região da Depressão Central do Rio Grande do Sul estende-se por planícies e relevos suaves ondulados, com predominio de florestas de galeria. Este estudo teve o objetivo de investigar a estrutura e a diversidade dos remanescentes de Floresta Estacional Decidual em diferentes posições na paisagem de pequenos tributários ao longo de uma microbacia em Pantano Grande, RS. Os levantamentos foram realizados em 40 pontos amostrais com $500 m^{2}$. Foi constatada a existência de três grupos ecológicos, espacialmente correlacionados à posição no relevo, tendo diferentes estágios de conservação, diversidade, estrutura e florística. O grupo 1, formação submontana (FS), destacou-se pela maior densidade de indivíduos nas maiores classes de diâmetro e pela maior altura, com alta dominância ecológica, determinada por uma particular espécie tolerante à sombra, Actinostemon concolor. O grupo 2, $F A / F S$, revelou-se com maior diversidade que os demais grupos, apresentando menor dominância ecológica. O grupo 3, formação aluvial (FA), foi caracterizado por indivíduos arbóreos menores e pela presença dominante de Sebastiania commersoniana.

Palavras-chave: ecologia, floresta subtropical, diversidade, solos florestais.

\section{ABSTRACT}

Central Depression region in the state of Rio Grande do Sul extends across plains and gentle undulated reliefs, with a predominance of gallery forests. This study aimed to analyze remaining tree communities of the seasonal deciduous forest in different positions of the landscape in small tributaries of a watershed in Pantano Grande, RS, Brazil. Surveys were conducted at 40 sampling points equaling $500 \mathrm{~m}^{2}$. The analysis pointed to the existence of three ecological groups, spatially correlated with their positions in the landscape, at different stages of preservation, diversity, structure and floristic development. The community groups were named sub-montane (upper plains) and alluvial formations, and the domain area of both formations (FA/FS). TheFA/FS proved to be more diverse than the others, with lower ecological dominance in narrow fragments. Submontane stood out with higher density of individuals in the largest diameter and height classes, with high ecological dominance, determined by a particular species tolerant to shade, Actinostemon concolor. Alluvial formation (FA) was characterized by smaller individual trees and the dominant presence of Sebastiania commersoniana in narrow corridors.

Key words: ecology, diversity, subtropical forest, forest soil.

\section{INTRODUÇÃO}

Em uma microbacia há uma gama desconcertante de fatores ecológicos que influenciam a sucessão florestal e os padrões fisionômicos e fitossociológicos das florestas. As condições ambientais e recursos disponíveis variam no espaço e no tempo (WIMBERLY \& SPIES, 2001). Os regimes de perturbação influenciam nos padrões espaciais e temporais e na mortalidade de árvores e proporcionam a criação de novos sítios para o estabelecimento de outros indivíduos arbóreos. A resposta da comunidade a esses fatores depende da história de vida das espécies componentes, bem como dos seus mecanismos de dispersão, taxas de crescimento e longevidade. Esses múltiplos fatores se combinam para conduzir a sucessão e produzir padrões de vegetação e

\footnotetext{
IUniversidade Federal de Santa Catarina (UFSC), 89520-000, Curitibanos, SC, Brasil. E-mail: marcelo.scipioni@gmail.com. Autor para correspondência.

"Departamento de Engenharia Florestal, Universidade Federal do Paraná (UFPR), Curitiba, PR, Brasil.

IIIDepartamento de Engenharia Florestal, Universidade Federal de Santa Maria (UFSM), Santa Maria, RS, Brasil.

${ }^{\text {IV }}$ Departamento de Ciência do Solo, UFSM, Santa Maria, RS, Brasil. 
diversidade na escala de paisagem (CONNELL, 1978; WIMBERLY \& SPIES, 2001).

A região da Depressão Central do Rio Grande do Sul apresenta-se em uma área de tensão ecológica entre o avanço recente das formações florestais nos últimos 3 a 5 mil anos sobre as áreas campestres do Sul do Brasil, favorecidas por mudanças climáticas para uma condição atualmente mais úmida e quente ( BEHLING et al., 2001). Essas florestas em pequenos tributários localizam-se em faixas com larguras variáveis, normalmente estreitas em relevo aluvial e, por vezes, sulcadas em pequenos vales ou em torrentes em áreas mais dissecadas em pequenas colinas, que representam respectivamente as formações, aluvial e submontana. Desse modo, ao longo da posição transversal e longitudinal dessas formações, uma ou mais comunidades podem ser distintas pela composição florística, sendo associadas às diferenças de solos e forma de relevo que condicionam aos diferentes déficits hídricos em uma escala local (MARCHIORI, 2004; SCIPIONI, 2012).

Assim sendo, o presente trabalho visa a contribuir na descrição da diversidade e estrutura dessas florestas remanescentes sobre diferentes condições pedológicas e geomorfológicas em gradiente ambiental, regulado por uma microbacia, abrangendo formações submontanas e aluviais. O propósito do trabalho é verificar diferenças entre as comunidades arbóreas, bem como as possíveis interações ambientais e os distúrbios na estruturação da floresta estacional decidual, vinculada aos pequenos cursos de água.

\section{MATERIAL E MÉTODOS}

Este estudo foi realizado no Rio Grande do Sul, em uma microbacia localizada na margem direita do baixo rio Jacuí, na região da Depressão Central. A área pertence à Estância Boa Vista e encontra-se entre os municípios de Pantano Grande e Rio Pardo. A geologia é formada por sedimentos paleozoicos que fazem parte do rebordo da bacia do Paraná, com predomínio de arenitos médios a finos, siltitos argilosos e lamitos de cores avermelhadas e brancas (SOARES et al., 2008). O clima da região caracteriza-se por temperatura média anual de $18,8^{\circ} \mathrm{C}$, variando de $18,1^{\circ} \mathrm{C}$ a $22^{\circ} \mathrm{C}$. A precipitação é em torno de $1400 \mathrm{~mm} \mathrm{ano}^{-1}$, tendo um balanço hídrico excedente de zero a $200 \mathrm{~mm}$ e deficiência entre 1 a $150 \mathrm{~mm}$, em curtos períodos de seca no verão. O clima é classificado como subtemperado úmido por MALUF (2000). As florestas da região são classificadas como Floresta Estacional Decidual Aluvial e Submontana (IBGE, 1986). A coleta de dados foi feita em 40 pontos amostrais permanentes de $500 \mathrm{~m}^{2}$ cada, distribuídos aleatoriamente ao longo do gradiente topográfico, acompanhando as torrentes e cursos de água, evitando as bordas dos fragmentos. Em cada ponto amostral, foram identificados e medidos todos os indivíduos lenhosos com diâmetro maior ou igual a $5,0 \mathrm{~cm}$ a $1,30 \mathrm{~m}$ de altura do solo (DAP) e determinadas às classes de solos. A altura das árvores foi estimada por comparação da extensão do cabo telescópico de um podão de poda alta. Os materiais botânicos dos indivíduos arbóreos não identificados foram submetidos a especialistas, sendo esses incorporados aos herbários do Departamento de Ciências Florestais da Universidade Federal de Santa Maria e da Escola de Florestas de Curitiba da Universidade Federal do Paraná. A nomenclatura botânica foi verificada pela Lista da Flora do Brasil (FORZZA et al., 2013).

Com auxílio do PC-ORD for Windows versão 6.0 (MCCUNE \& MEFFORD, 2011), foi efetuada a Análise de Correspondência Retificada (DCA) para verificação da existência de grupos florísticos na área. Os resultados foram originados por uma matriz de dados, composta pela densidade de todas as espécies por ponto amostral e transformados os dados de abundância por $\log (n+1)$. As parcelas foram categorizadas por suas respectivas classes de solos (EMBRAPA, 2013). Para cada grupo evidenciado por este procedimento, foram calculados os parâmetros fitossociológicos (MUELLERDOMBOIS \& ELLEMBERG, 2002). Os parâmetros densidade absoluta e área basal foram computados por ponto amostral e foram comparados por meio da ANOVA unifatorial, associada ao teste de Tukey. A frequência da distribuição da densidade dos indivíduos por classes de diâmetro e altura para os grupos foram analisados conforme FELFILI \& REZENDE (2003). Após, as classes foram comparadas pelo teste QuiQuadrado de partição para verificar a existência de diferenças entre os fragmentos, sendo os dados processados pelo software BioEstat 5.0 (AYRES et al., 2007). Os índices de diversidade de Shannon, Equabilidade de Pielou, Simpson e Dominância, com os seus respectivos intervalos de confiança (95\%), foram obtidos, assim como os estimadores de riqueza Jackknife de $1^{\mathrm{a}}$ e $2^{\mathrm{a}}$ ordem. As comparações dos índices de diversidade foram realizadas aos pares por randomização das amostras pelo teste bootstrap, com obtenção do valor de $p$. Para essa análise, foi utilizado o mesmo número de pontos amostrais por grupo. Esses dados foram computados pelo software Past, versão 2.14,conforme HAMMER et al. (2012).

\section{RESULTADOS E DISCUSSÃO}

Como resultado da ordenação florística, destacaram-se três agrupamentos, que estão 
espacialmente setorizados no ambiente em cotas altimétricas inferiores $(<50 \mathrm{~m})$, intermediárias $(50-70 \mathrm{~m})$ e superiores $(>70 \mathrm{~m})$, correlacionados ao primeiro eixo, com elevado autovalor $(0,659 ; \mathrm{P}=0,001)$. O segundo eixo obteve baixo autovalor $(0,234, \mathrm{P}=0,935)$, que expressa baixa significância na ordenação. Assim, esse resultado evidencia, pela alternância na abundância das espécies, a existência de um gradiente, conforme TERBRAAK (1995), formado ao longo da encosta e da planície dos tributários do baixo rio Jacuí. Os pares de eixos 1 vs 2 apresentaram maior $\mathrm{R}^{2}(0,528)$, com melhor representação dos agrupamentos e do gradiente ambiental. Na figura 1, nota-se alta proximidade entre os pontos amostrais do grupo 1, condicionado pela

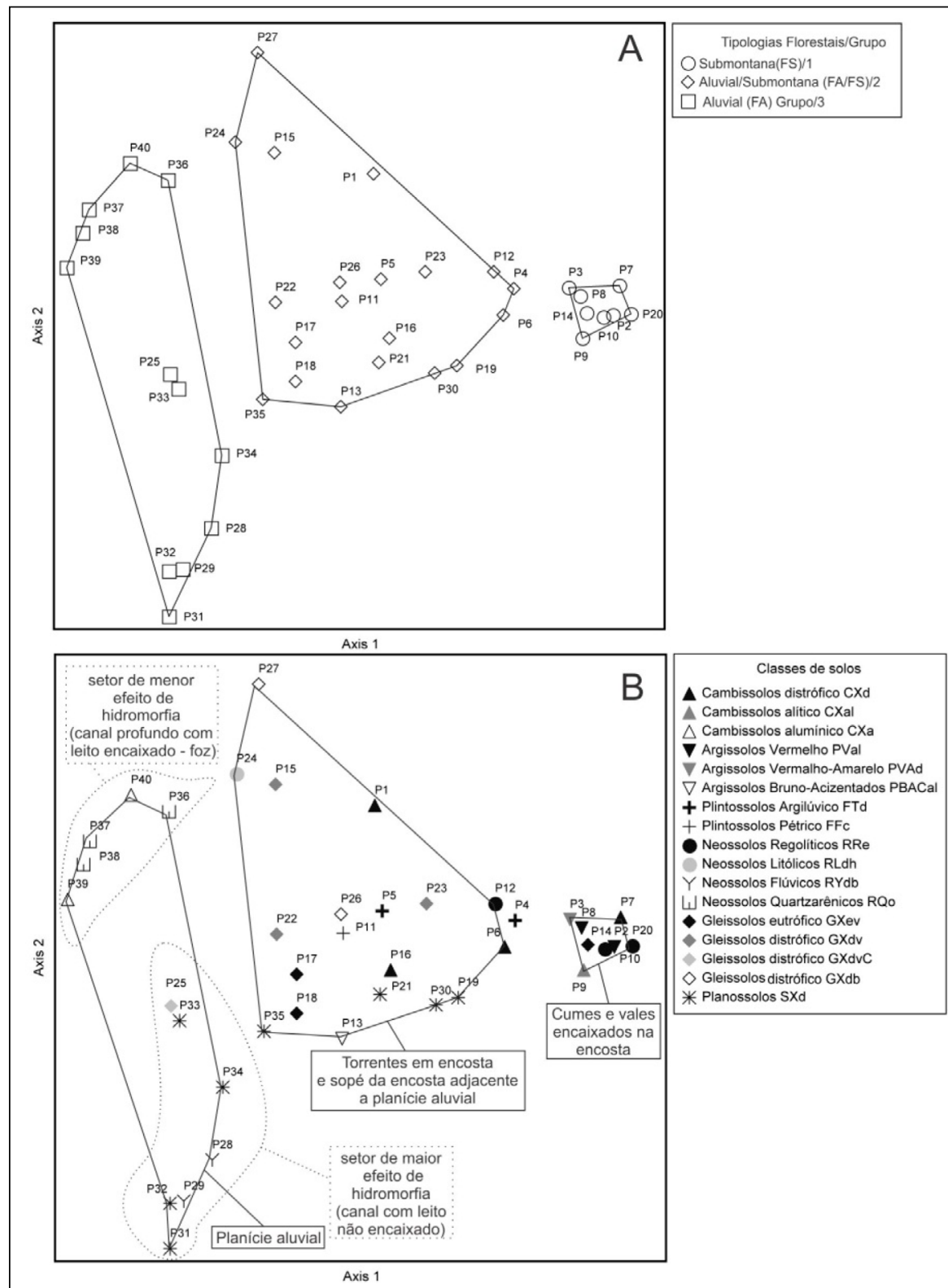

Figura 1 - Análise de Correspondência Retificada com representação da classificação dos pontos amostrais por grupos florísticos: $(\mathrm{A})$ correlacionados às tipologias florestais e $(\mathrm{B})$ os pontos amostrais categorizados por classe de solos, Pantano Grande, RS. Simbologia do Sistema Brasileiro de Classificação de Solos (Embrapa, 2013) 1ํnível: na legenda da figura em conjunto com o atributo de distinção entre os solos de mesma classe em primeiro nível. 2ำ nível: X: Háplico, V: Vermelho, VA: Vermelho-amarelo, BAC: Bruno-acinzentado, T: Argilúvico, F: Pétrico, R: Regolítico, L: Litólico, Y: Flúvico, Q: Quartzarênico. 3o e 4nível: d: distrófico, al: alítico, a: alumínico, c: concrecionário, e: eutrófico, dh: distroúmbrico, o: órtico, b: argila de atividade baixa, v: argila de atividade alta. C: cambissólico.

Ciência Rural, v.45, n.10, out, 2015. 
alta dominância florística, com pontos de ocorrência exclusiva na encosta, sendo assim, definido como formação submontana. Nos agrupamentos 2 e 3, a ordenação apresentou uma maior espacialização dos pontos amostrais, acondicionados pelo segundo eixo, por apresentarem maiores variações na composição florística e nas condições de solos. O grupo 2, considerado como área de ambas as formações FS/ FA, foi em razão de apresentar pontos em torrentes na encosta e no sopé delas; e o grupo 3, aluvial, por pontos exclusivos na planície, com predomínio em solos hidromórficos em cota inferior. Os Gleissolos foram agrupados no grupo 2, por não terem caráter aluvial.

No grupo 1, Actinostemon concolor (laranjeira-do-mato) foi a mais importante em razão de sua alta densidade e frequência, representando um valor de percentual de importância (PI) de 21,7\%. A diversidade desse grupo foi baixa em razão da dominância ecológica de Actinostemon concolor, espécie climácica tolerante à sombra, favorecida pelo ambiente sombreado em terreno com vale encaixado. A fisionomia do grupo é típica da formação submontana (FS), sendo caracterizado pela predominância de Actinostemon concolor e Sorocea bonplandii $(4,7)$ no sub-bosque, associado com indivíduos de maior porte, com destaque para as espécies: Luehea divaricata (5,9\%), Cordia americana (5,7\%), Chrysophyllum marginatum (5,6\%) e Myrcianthes pungens $(5,2 \%)$. A área basal foi elevada com uma estimativa de $40,0 \mathrm{~m}^{2} \mathrm{ha}^{-1}$, que, segundo LONGHI (1997), caracteriza estágios secundários avançados com melhor grau de conservação da floresta. Esse resultado fitossociológico de sub-bosque com alto percentual de importância para espécie Actinostemon concolor e alto valor de área basal é semelhante aos encontrados por JARENKOW \& WAECHTER (2001), JURINITZ \& JARENKOW (2003) e SCIPIONI et al. (2011).

No grupo 2, as espécies em ordem de porcentagem de importância que se destacaram no grupo foram Chrysophyllum marginatum (7,4\%), Luehea divaricata (5,5\%), Sebastiania brasiliensis (5,4\%), Casearia sylvestris $\quad(4,9, \%), \quad$ Lithrea brasiliensis (4,7\%) e Sebastiania commersoniana $(4,6 \%)$. Esse grupo apresentou baixa dominância ecológica, resultando em um maior valor no índice de diversidade, potencializada pela maior amplitude geográfica e variabilidade ambiental. O grupo 2 apresenta traços florísticos e ambientais de ambas as formações, aluvial e submontana, sendo assim considerada como FA/FS, em áreas de interflúvios com fragmentos florestais estreitos, possibilitando a presença de espécies de ambos os grupos, aluvial e submontano, sem dominância ecológica de espécies típicas dessas formações.

O grupo 3, na planície sobre os tributários da microbacia, caracteriza a formação aluvial (FA) por causa da alta densidade (637 indivíduos ha ${ }^{-1}$ ) e frequência (100\%) de Sebastiania commersoniana, percentual de importância de 19,5\%. Essa situação está de acordo com os estudos fitossociológicos em florestas às margens de grandes rios da região (BUDKE et al., 2007; MARCHI \& JARENKOW, 2008), assim como em estudos de outras unidades fitogeográficas no Sul do Brasil em áreas aluviais (BARDDAL et al., 2004; NOGUEIRA et al., 2010). As variações pedológicas e hídricas dos solos na planície resultaram na separação de dois subgrupos. No ambiente de maior saturação hídrica, destacou-se Sebastiania commersoniana $(\mathrm{PI}=35,2 \%)$ e, no outro subgrupo com menor efeito de hidromorfia, houve um decréscimo de sua contribuição $(\mathrm{PI}=8,5 \%)$, com realce na importância de Eugenia uniflora (7,8\%) e Luehea divaricata (9,9\%). Resultado semelhante para a espécie Sebastiania commersoniana foi observado por NOGUEIRA et al. (2010), caracterizando a fitossociologia florestal em compartimentos pedológicos com diferentes níveis de hidromorfia.

Analisando os três grupos, a densidade de árvores por área não apresentou diferenças significativas entre os grupos, isso em razão dos altos valores dos desvios padrão $\left(\mathrm{F}_{0,10}=1,628 ; \mathrm{P}=0,210\right)$. Essas variações de densidade se devem aos diferentes ambientes e estágios sucessionais presentes na microbacia, condicionados por distúrbios naturais e antrópicos. Por outro lado, analisando-se cada grupo separadamente, verificaram-se níveis distintos de variação estrutural para área basal, além das diferenças de riqueza e diversidade em relação ao conjunto das áreas. O intervalo estimado para a riqueza específica, esperada para toda a comunidade arbórea, variou entre 108,5 e 113,6 espécies, segundo os estimadores Jackknife de $1^{\underline{a}}$ e $2^{2}$ ordem. Dessa forma, a riqueza inventariada estaria representando entre $83,6 \%$ a $87,5 \%$ das espécies esperadas por esses índices (Tabela 1).

$\mathrm{Na}$ tabela 2, todas as comparações entre os índices de diversidade entre os grupos foram significativamente diferentes $(\mathrm{p}<0,001)$ pelo teste de bootstrap. O grupo 2 apresentou maior diversidade em comparação com os demais grupos, em razão da sua localização predominantemente entre as formações, com maior amplitude geográfica e contribuição florística das tipologias dos grupos $1 \mathrm{e}$ 3. Esses dois grupos apresentaram alta dominância específica, respectivamente, por pequeno número 
Tabela 1 - Estimativas dos parâmetros fitossociológicos, riqueza e diversidade para a sinúsia arbórea nos grupos e amostra geral, Pantano Grande, RS.

\begin{tabular}{|c|c|c|c|c|c|c|}
\hline Variáveis & Grupo $1(N=8 ; 0,4$ ha $)$ & Grupo $2(N=20 ; 1$ ha $)$ & Grupo 3 ( $N=12 ; 0,6$ ha $)$ & Amostra total $(N=40)$ & $F$ & $P$ \\
\hline $\mathrm{N}$ & 723 & 1733 & 1301 & 3757 & - & - \\
\hline DA (ind. ha ${ }^{-1}$ ) & $1.807 \pm 286$ & $1.733 \pm 590$ & $2.168 \pm 930$ & $1.878,5$ & 1,62 & 0,21 \\
\hline $\operatorname{DoA}\left(\mathrm{m}^{2} h \mathrm{~h}^{-1}\right)$ & $40,0 \pm 11,6^{\mathrm{a}}$ & $35,9 \pm 7,8^{\mathrm{ab}}$ & $31,9 \pm 10,1^{\mathrm{b}}$ & 35,3 & 2,08 & 0,14 \\
\hline Mortos VA (indha ${ }^{-1}$ ) & $57,5 \pm 51,7$ & $84,0 \pm 62,1$ & $96,6 \pm 64,8$ & $82,5 \pm 61,1$ & 0,997 & 0,379 \\
\hline $\mathrm{H}^{\prime}$ & 2,29 & 3,62 & 2,74 & 3,52 & - & - \\
\hline $\mathrm{J}$ & 0,60 & 0,82 & 0,69 & 0,77 & - & - \\
\hline Famílias & 24 & 33 & 23 & 37 & - & - \\
\hline Gêneros & 39 & 61 & 44 & 67 & - & - \\
\hline Espécies (spp.) & 50 & 83 & 52 & 95 & - & - \\
\hline S (Jackknife1) & 56,37 & 98,05 & 68,5 & 108,55 & - & - \\
\hline S (Jackknife2) & 56,48 & 106,62 & 78,2 & 113,61 & - & - \\
\hline
\end{tabular}

Em DoA, DA e VA: as diferenças de letras evidenciaram diferenças estatísticas significantes ao nível=0,01 e os valores sem letras foram não significativos. Siglas: $N=$ unidades amostrais; $\mathrm{N}=$ número de indivíduos; $\mathrm{DA}=$ densidade absoluta; DoA $=$ dominância absoluta; $\mathrm{VA}=$ valor absoluto de indivíduos mortos em pé; spp. = quantidade de espécies; $\mathrm{H}^{\prime}=$ índice de Shannon; J' = equabilidade de Pielou; $\mathrm{S}=$ estimador de riqueza de espécies de Jacknife $1^{\mathrm{a}}$ e $2^{\mathrm{a}}$ ordem.

de espécies típicas do sub-bosque clímax tolerantes à sombra e reduzido número de espécies adaptadas ao ambiente aluvial, contribuindo para diminuição da diversidade de ambas as situações.

$\mathrm{Na}$ análise de densidade por classes de diâmetros entre os grupos, houve diferença significativa em todas as classes $\left(\chi_{0,05}^{2} ;{ }_{12}=207,2\right.$; $\mathrm{P}<0,0001)$. Nas menores classes, com diâmetro de 4,0 a 20,0 cm, o grupo 3 apresentou maior densidade de indivíduos, quando comparada com os demais grupos $\left(\chi_{0,05}^{2} ;=10,43 ; \mathrm{P}<0,0012\right)$. Entre as classes intermediárias, de 20 a $28 \mathrm{~cm}$ de diâmetro, o grupo 2 apresentou maior densidade relativa, em comparação com os demais $\left(\chi_{0,05 ; 4}^{2}=7,70 ; \mathrm{P}<0,0055\right)$. A partir das classes de maiores dimensões, acima de $28 \mathrm{~cm}$ de diâmetro, o grupo 1 destacou-se dos demais $\left(\chi_{0,05 ; 6}^{2}=47,38 ; \quad \mathrm{P}<0,0001\right), \quad$ principalmente em comparação com ao grupo 3, que apresentou apenas 3 a 5 indivíduos por hectare nas classes acima de $44 \mathrm{~cm}$ de diâmetro. Esses valores são muito inferiores aos 8 a 15 ind. ha ${ }^{-1}$ do grupo 1 e aos 7 a 14 ind. ha ${ }^{-1}$ do grupo 2. Constatou-se, dessa forma, que os grupos $1 \mathrm{e}$ 2 apresentam maiores concentrações de indivíduos de maior porte. A distribuição dos indivíduos em classes de altura revelou, para o grupo 3 , que 525ind. ha ${ }^{-1}$ concentram-se na altura entre 2 a $8 \mathrm{~m}$, enquanto, nessa mesma classe, este valor correspondeu a 418 ind. ha ${ }^{-1}$ e 308ind. ha ${ }^{-1}$, respectivamente, nos grupos 1 e 2 . No grupo 3, foram verificados apenas 3 indivíduos (5ind. $\mathrm{ha}^{-1}$ ) com altura superior a $16 \mathrm{~m}$, com destaque para as espécies Sebastiania commersoniana (1) e Salix humboldtiana (2). Nos demais grupos, o maior número proporcional de árvores por hectare foi amostrado com altura superior a $16 \mathrm{~m}$, sendo $87,5 \mathrm{ind}$. ha $^{-1}$ no grupo 1 e 36 ind. ha-1 no grupo 2 . A distribuição de frequência dos indivíduos por classes de altura entre os trechos evidenciou diferenças significativas $\left(\chi_{0,05 ; 18}^{2}=483,93 ; \quad \mathrm{P}<0,0001\right), \quad$ proporcionadas

Tabela 2 - Índices de diversidade dos grupos florísticos e seus respectivos valores de intervalo de confiança 95\%. Estância Boa Vista, Pantano Grande, RS.

\begin{tabular}{|c|c|c|c|c|}
\hline Diversidade & Grupo $1-\mathrm{FS}(N=8)$ & Grupo 2 - FA/FS $(N=8)$ & Grupo 3 - FA $(N=8)$ & valor de $\mathrm{P}$ \\
\hline $\mathrm{N}$ & 723 & 665 & 956 & - \\
\hline Spp. & 50 & 61 & 40 & - \\
\hline $\mathrm{H}^{\prime}$ & $2,29(2,12$ a 2,39$)$ & $3,42(3,29$ a 3,45$)$ & $2,58(2,48$ a 2,65$)$ & 0,001 \\
\hline $\mathrm{J}$ & $0,60(0,56$ a 0,63$)$ & $0,83(0,82$ a 0,86$)$ & $0,70(0,69$ a 0,79$)$ & 0,001 \\
\hline Simpson & $0,72(0,68$ a 0,75$)$ & $0,95(0,94$ a 0,96$)$ & $0,86(0,84$ a 0,87$)$ & 0,001 \\
\hline Dominância & $0,27(0,24$ a 0,31$)$ & $0,046(0,043$ a 0,052$)$ & $0,14(0,12$ a 0,15$)$ & 0,001 \\
\hline
\end{tabular}

Siglas: $N=$ unidades amostrais; $\mathrm{N}=$ número de indivíduos; Spp.=quantidade de espécies; $\mathrm{H}^{\prime}=$ índice de Shannon; J'=equabilidade de Pielou; Índice de dominância=1- índice de Simpson. 
principalmente pelas maiores densidades observadas de árvores com altura entre 4 a $6 \mathrm{~m}$, no grupo 3, e com altura entre 14 a $18 \mathrm{~m}$, no grupo 1 . Também foram verificadas densidades inferiores à esperada de árvores na primeira classe de altura e de indivíduos com altura superior a $10 \mathrm{~m}$, no grupo 3 .

\section{CONCLUSÃO}

$\mathrm{Na}$ análise de ordenação, foi possível diferenciar três grupos florísticos, formação submontana, aluvial e área com ambas as formações (FA/FS), condicionadas por diferenças florísticas e fitossociológicas, com certa adequação na paisagem. A formação submontana destacou-se pela maior densidade de indivíduos nas maiores classes de diâmetro e pela maior altura, com alta dominância ecológica por uma espécie tolerante à sombra, $\boldsymbol{A}$. concolor, concentrando-se em pequenos vales. As áreas com ambas as formações revelaram-se com maior diversidade que os demais grupos, apresentando menor dominância ecológica em razão da contribuição das tipologias localizadas nos extremos do gradiente. A posição de interflúvio da microbacia abrangeu a maioria dos pontos amostrais dessa situação, favorecendo a expansão dos componentes aluviais e submontanos. A formação aluvial, na planície, foi caracterizada por indivíduos arbóreos menores e pela presença dominante de $\boldsymbol{S}$. commersoniana.

\section{AGRADECIMENTOS}

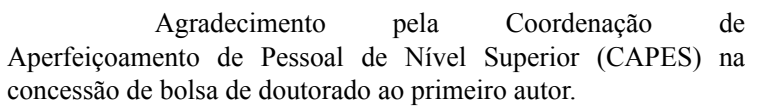

\section{REFERÊNCIAS}

AYRES, M. et al. BioEstat: aplicações estatísticas nas áreas das ciências bio-médicas. Belém: 2007. Versão 5.0.

BARDDAL, M.C. Caracterização florística e fitossociológica de um trecho sazonalmente inundável de floresta aluvial, em Araucária, PR. Ciência Florestal, v.14, n.2, p.37-50, 2004.

BEHLING, H. et al. Holocene environmental changes in the São Francisco de Paula region, southrn Brazil. Journal of south American Earth Sciences, v.14, p.631-639, 2001. Disponível em: $<$ http://www.sciencedirect.com/science/journal/08959811/14/6>. Acesso em: 10 jan. 2011. doi: 10.1016/S0895-9811(01)00040-2.

BUDKE, J.C. et al. Relationships between tree component structure, topography and soils of a riverine forest, Rio Botucaraí, southern Brazil. Plant Ecol, v.189, p.187-200, 2007. Disponível em: <http:// www.icb.ufmg.br/treeatlan/Downloads/a104.pdf $>$. Acesso em: 10 jan. 2012. doi: 10.1007/s11258-006-9174-8.

CONNELL, J.H. Diversity in tropical rain forest and coral reefs. Science, v.199, n.4335, p.1302-1310, 1978. Disponível em: <http://www.jstor.org/stable/1745369>. Acesso em: 10 jan. 2012. doi: $10.2307 / 1745369$.

EMBRAPA (EMPRESA BRASILEIRA DE PESQUISA AGROPECUÁRIA), Centro Nacional de Pesquisa de Solos. Sistema de classificação brasileiro de classificação de solos. 3.ed. Rio de Janeiro, 2013. 353p.

FELFILI, J.M.; REZENDE, R.P. Conceitos e métodos em fitossociologia. Brasília: UNB, 2003. 68p. (Comunicações técnicas florestais, 1).

FORZZA et al. Lista da flora do Brasil. Jardim Botânico do Rio de Janeiro. Disponível em: <http://reflora.jbrj.gov.br/jabot/ PrincipalUC/PrincipalUC.do>. Acesso em: 09 out. 2013. Online.

HAMMER, Ø et al. Past: paleontological statistics software package for education and data analysis. versão 2.14. Palaeontologia Electronica, v.4, n.1, p.1-9, 2012.

IBGE (FUNDAÇÃO INSTITUTO BRASILEIRO DE GEOGRAFIA E ESTATÍSTICA). Folha SH.22 Porto Alegre e partes das Folhas SH. 21 Uruguaiana e SI. 22 Lagoa Mirim: geologia, geomorfologia, pedologia, vegetação, uso potencial da terra. Rio de Janeiro: IBGE, 1986. (Levantamento dos Recursos Naturais, v.33).

JARENKOW, J.A.; WAECHTER, J. Composição, estrutura e relações florísticas do componente arbóreo de uma floresta estacional no Rio Grande do Sul, Brasil. Revista Brassileira Botânica, v.24, n.3, p.263-272, 2001.

JURINITZ, C.F.; JARENKOW, J.A. Estrutura do componente arbóreo de uma floresta estacional na Serra do Sudeste, Rio Grande do Sul, Brasil. Revista Brasileira de Botânica, v.26, n.4, p.475-487, 2003.

LONGHI, S.J. Agrupamento e análise fitossociológica de comunidades florestais na sub-bacia hidrográfica do rio Passo Fundo-RS. 1997.198f. Tese (Doutorado em Engenharia Florestal) - Curso de Pósgraduação em Engenharia Florestal, Universidade Federal do Paraná, PR.

MALUF, J.R.T. Nova classificação climática do Estado do Rio Grande do Sul. Revista Brasileira de Agrometeorologia, v.8, n.1, p.141-150, 2000.

MARCHI, T.C. de; JARENKOW, J.A. Estrutura do componente arbóreo de mata ribeirinha no rio Camaquã, município de Cristal, Rio Grande do Sul, Brasil. Iheringia, Sér. Bot., v.63, n.2, p.241-248, 2008.

MARCHIORI, J.N.C. Fitogeografia do Rio Grande do Sul: Campos sulinos. Porto Alegre: EST Edições, 2004. 110p.

MCCUNE, B.; MEFFORD M.J. PC-ORD: multivariate analysis of ecological data. Gleneden Beach: MjM Software, 2011.Version 6.0.

MUELLER-DOMBOIS, D.; ELEMBERG, H. Aims and methods of vegetation ecology. 2.ed.New Jersey: The Blackburn, 2002. 547p.

NOGUEIRA, M.K.F. et al. Caracterização fitossociológica e pedológica dos estratos arbóreo e arbustivo em uma planíce aluvial no município de Jaguariaíva, Paraná. Terr@Plural, v.4, n.2, p.193-215, 2010.

SCIPIONI, M.C. et al. Fitossociologia em fragmento florestal no noroeste do Estado do Rio Grande do Sul. Ciência Florestal, v.21, p.407-417, 2011. 
SCIPIONI, M.C. et al. Análise fitossociológica de um fragmento de floresta estacional em uma catena de solos no Morro do Cerrito, RS. Ciência Florestal, v.22, n.3, p.467-476, 2012. Disponível em: <http://cascavel.ufsm.br/revistas/ojs-2.2.2/index.php/ cienciaflorestal/article/view/6614>. Acesso em: 22 fev. 2013. doi: 10.5902/198050986614.

SOARES, A.P. et al. Correlações estratigráficas conflitantes no limite Permo-Triássico no Sul da Bacia do Paraná: O Contato entre duas sequências e implicações na configuração espacial do aquífero Guarani. Revista Pesquisas em Geociências, v.35, n.2, p.115-133, 2008.

TER BRAAK, C.J.F. Ordination. In: JONGMAN, R.H.G.et al. Data analysis in community and landscape ecology. Cambridge: Cambridge University, 1995. 299p.

WIMBERLY, M.C.; SPIES, T.A. Influences of environment and disturbance on forest patterns in coastal Oregon watersheds. Ecology, v.82, n.5, p.1443-1459, 2001. Disponível em: <http:// www.jstor.org/stable/2680001>. Acesso em: 22 jul. 2011. 